\title{
Study of Intelligent Photovoltaic System Fault Diagnostic Scheme Based on Chaotic Signal Synchronization
}

\author{
Chin-Tsung Hsieh and Jen Shiu \\ Department of Electrical Engineering, National Chin-Yi University of Technology, No. 57, Section 2, Zhongshan Road, \\ Taiping District, Taichung 41170, Taiwan \\ Correspondence should be addressed to Chin-Tsung Hsieh; fred@ncut.edu.tw
}

Received 14 July 2013; Revised 10 September 2013; Accepted 18 September 2013

Academic Editor: Dumitru Baleanu

Copyright (c) 2013 C.-T. Hsieh and J. Shiu. This is an open access article distributed under the Creative Commons Attribution License, which permits unrestricted use, distribution, and reproduction in any medium, provided the original work is properly cited.

\begin{abstract}
As the photovoltaic system consists of many equipment components, manual inspection will be very costly. This study proposes the photovoltaic system fault diagnosis based on chaotic signal synchronization. First, MATLAB was used to simulate the fault conditions of solar system, and the maximum power point tracking (MPPT) was used to ensure the system's stable power and capture and record the system fault feature signals. The dynamic errors of various fault signals were extracted by chaotic signal synchronization, and the dynamic error data of various fault signals were recorded completely. In the photovoltaic system, the captured output voltage signal was used as the characteristic values for fault recognition, and the extension theory was used to create the joint domain and classical domain of various fault conditions according to the collected feature data. The matter-element model of extension engineering was constructed. Finally, the whole fault diagnosis system is only needed to capture the voltage signal of the solar photovoltaic system, so as to know the exact fault condition effectively and rapidly. The proposed fault diagnostor can be implemented by embedded system and be combined with ZigBee wireless network module in the future, thus reducing labor cost and building a complete portable renewable energy system fault diagnostor.
\end{abstract}

\section{Introduction}

The rapid development of technology has led to over dependence on energy. Although oil is the most important energy source at present, it will be in shortage in the next 50 years. In order to solve this problem, humans have begun to seek alternative energy sources which will not harm the earth, such as renewable energy sources. The renewable energy sources are the energy sources derived from the nature, such as solar energy, wind power, tidal power, and geothermal heat, which are inexhaustible energy sources. Fossil energy is exhaustible, sunlight can meet 2,850 times of global energy needs, wind energy can meet 200 times of global energy needs, hydraulic power can meet 3 times of global energy needs, biomass energy can meet 20 times of global energy needs, and geothermal heat can meet 5 times of global energy needs.

This study focused on solar photovoltaic power generation. In the regions with abundant light supply (e.g., space exposed to the sun, ocean, coast, and open rocky area), solar energy is supplied continuously, and the production process does not pollute the environment or consume other earth resources or cause greenhouse effect. Solar energy is derived from the sun inexhaustibly and is as good as water power or wind power. Many countries are developing solar energy actively. The solar energy facilities can be constructed facilities, such as wind energy facilities, protecting many lands and ecologies. The solar module can be mounted on buildings, which is called photovoltaic integrated buildings. The solar panel can generates electric power in sunlight, insulate heat, reduce the temperature inside buildings effectively, and reduce the building energy consumption. Solar energy is a renewable energy that can be conveniently obtained. As long as the solar panel is placed in an open area with long sunshine, the solar energy can be obtained effectively. However, as the equipment is set in open area for a long time, it is likely to be destroyed by natural disasters and to age. These factors will reduce the efficiency of solar photovoltaic system. Once a fault occurs in the solar module, it must be inspected, but the 


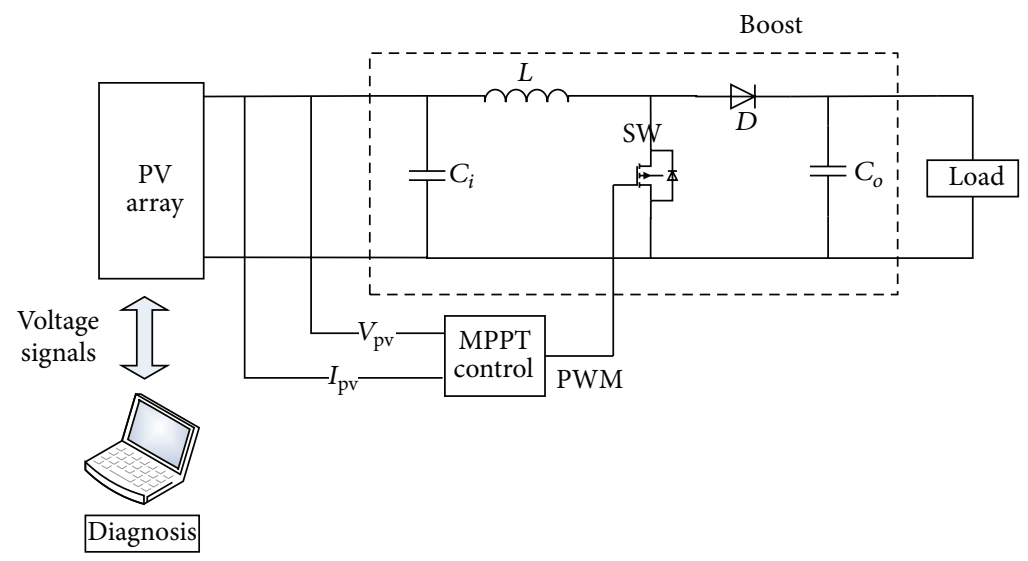

FIGURE 1: Solar photovoltaic system structure.

faults may be difficult to detect with naked eye. The detection is labor and cost consuming. This study thus proposes an intelligent solar photovoltaic system fault diagnostor. When small solar power systems are popularized and combined with commercial power, the household solar power system is likely to have faults; thus, the solar photovoltaic system fault diagnostor will be an important tool. The research and development of solar energy fault diagnostor will be very helpful to solar power systems, not only shortening manual detection time and reducing risks but also detecting the fault point effectively.

Most current studies on the fault diagnosis for solar photovoltaic system use software simulated or measured power generation data of solar photovoltaic system as the fault diagnosis data. As the output power of solar photovoltaic system is influenced by the sunshine intensity and module surface temperature significantly and the solar power system data are nonlinear system, many studies use software for simulation. Several diagnostic methods for solar photovoltaic system are introduced as follows. Literature [1] proposed a diagnostic method based on error backpropagation algorithm (BP), combined with the unique characteristics of calculator and artificial neural network, such as rapid development of complexity, association, memory, adaptability, self-learning, and processing complex multimode. The artificial neural network $[1,2]$ is applicable to fault diagnosis, and BP neural network is the most used one. The input signal is transmitted from the input layer and hidden layer to the output layer. The output end generates the output signal. The weight of network is fixed in initial propagation, and the state of neuron of a layer only influences the state of neuron of next layer. When the output signal is not as good as the output layer expected, the error backpropagation is triggered. In the error signal backpropagation, the weight of network can adjust the error signal. However, the defect in neural network is that the optimal number of neurons of hidden layer should be selected through multiple experiments, and a long training is required in order to converge to the system. Literature [3] used MATLAB software to simulate the characteristics of solar photovoltaic system. Equivalent mathematical equation was used to simulate the characteristic curves of photovoltaic system in sunlight and in shadow. The simulation system sets the series-parallel connection of modules and photovoltaic module shadow coverage area, whether or not to mount bypass diode and blocking diode. The simulation effect was very close to the actual result, but the equivalent parameter changed when a fault occurred. Thus, the defect in the above method is that the fault parameter is difficult to be calculated when the photovoltaic system has other faults. Literature $[4,5]$ used PSIM software to build a solar photovoltaic system. The characteristic curve of simulation result was similar to the actual operation curve, and the extension theory was applied to diagnose the fault category, so the diagnosis effect was very good. However, the curve of simulated photovoltaic system fault in $[4,5]$ was slightly different from the actual faulty system. The simulation system did not consider the influence of bypass diode and blocking diode on the photovoltaic system, and the actual photovoltaic system was equipped with bypass diode and blocking diode. Therefore, there was a pit in IV curve when the photovoltaic system had a fault, so that the maximum power point shifted, resulting in multiple peaks of PV characteristic curve. This paper proposes a fault diagnosis method based on chaotic synchronization signal, combined with the intelligent classification of extension theory. The chaotic system is very sensitive to the change in system parameter, and the system parameter of system must be changed if the solar photovoltaic system has a fault. Therefore, this method only needs to import the signal of the photovoltaic system with faults into the chaotic synchronization system according to the variance in system parameter and to capture the variance in dynamic error. It is imported into the extension theory to distinguish the fault state effectively, and the extension theory does not need learning time, so the diagnosis is rapid.

\section{Photovoltaic System}

The main function of solar photovoltaic system is to convert the solar irradiation into electrical energy by solar photovoltaic cell and an electric power converter. The equipped 


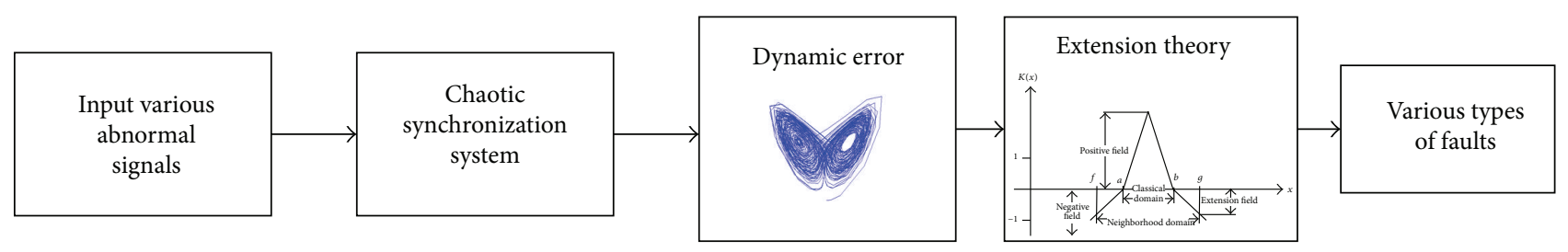

FIGURE 2: Chaotic signal synchronization and extension diagnostic system flow chart.

electric power converter can stabilize, increase and reduce the output voltage, or convert the frequency according to the requirement of output load. The output power depends on the area of solar photovoltaic cell, conversion efficiency, solar illumination, ambient temperature, and effect of load. The solar photovoltaic array used in this study was a $10 * 2$ array, equipped with a boost converter and MPPT algorithm, as shown in Figure 1. The MPPT algorithm guarantees the maximum power output when the solar photovoltaic array has faults. The voltage variation is measured in the system operation to distinguish the fault.

The power characteristic generated by solar photovoltaic cell is not linear, and the power of solar photovoltaic cell varies with current sunshine intensity and ambient temperature. In order to keep the output power at the maximum value of the characteristic curve [6], the solar photovoltaic system must be equipped with MPPT control to guarantee the maximum output power, so as to maximize the system output efficiency. At present, the common incremental conductance MPPT method [7] is used in this paper.

\section{Proposed Fault Diagnosis Method}

3.1. Chaotic Synchronization System. As the chaotic system is very sensitive to the change in system parameter [8-14], this study used this characteristic to capture the solar photovoltaic system fault voltage signal for chaotic synchronization transformation. The kinematic trajectories of dynamic error were extracted, and these kinematic trajectories were the fault features; thus, the extension theory was used to recognize fault conditions. The Lorenz chaotic synchronization system is expressed as follows [8]:

$$
\begin{aligned}
& \text { Master: }\left\{\begin{array}{l}
\dot{x}_{1}=\alpha\left(x_{2}-x_{1}\right), \\
\dot{x}_{2}=\beta x_{1}-x_{1} x_{3}-x_{2}, \\
\dot{x}_{3}=x_{1} x_{2}-\gamma x_{3},
\end{array}\right. \\
& \text { Slave: }\left\{\begin{array}{l}
\dot{y}_{1}=\alpha\left(y_{2}-y_{1}\right), \\
\dot{y}_{2}=\beta y_{1}-y_{1} y_{3}-y_{2}, \\
\dot{y}_{3}=y_{1} y_{2}-\gamma y_{3} .
\end{array}\right.
\end{aligned}
$$

Dynamic error of system is as follows:

$$
\left[\begin{array}{l}
\dot{e}_{1} \\
\dot{e}_{2} \\
\dot{e}_{3}
\end{array}\right]=\left[\begin{array}{ccc}
-\alpha & \alpha & 0 \\
\beta & -1 & 0 \\
0 & 0 & -\gamma
\end{array}\right]\left[\begin{array}{l}
e_{1} \\
e_{2} \\
e_{3}
\end{array}\right]+\left[\begin{array}{c}
0 \\
-y_{1} y_{3}+x_{1} x_{3} \\
y_{1} y_{2}-x_{1} x_{2}
\end{array}\right] \text {, }
$$

TABLE 1: Solar photovoltaic system fault category.

\begin{tabular}{ll}
\hline Fault category & Fault conditions \\
\hline PF1 & Normal operation \\
PF2 & Two series branches, one fault in any branch \\
PF3 & Two series branches, two faults in any branch \\
PF4 & Two series branches, three faults in any branch \\
PF5 & Two series branches, one fault in each branch \\
PF6 & Two series branches, two faults in each branch \\
PF7 & Two series branches, one fault in one branch, \\
& two faults in the other branch \\
PF8 & Two series branches, one fault in one branch, \\
& three faults in the other branch \\
PF9 & Two series branches, two faults in one branch, \\
& three faults in the other branch
\end{tabular}

where $\alpha, \beta$, and $\gamma$ are positive constants. According to the condition of chaos theory [8], if the eigenvalues of system are negative, the system state is determined as stable, so that the chaotic attractor can be generated. The kinematic trajectory of chaotic attractor is used for various studies. This paper observes the kinematic trajectory of chaotic attractor to distinguish the fault type of solar photovoltaic system.

3.2. Extension Theory. The extension is the real number extended from fuzzy set range $[0,1]$ to $[-\infty, \infty]$, representing the degree of a property of things. First, the maximum and minimum extents of characteristics of real number are determined. The maximum extent is set as $a^{\prime}$ and the minimum extent is $b^{\prime}$, and the real domain $\left[a^{\prime}, b^{\prime}\right]$ is obtained. The classical domain range $[a, b]$ can be worked out by (4) and (5), where $\alpha$ is the extend factor of classical domain, aiming at extension considering light error resulted from human or irresistible factors in actual measurement. This region is called classical domain [15].

Consider the following:

$$
\begin{array}{ll}
a=(1-\alpha) a^{\prime}, & b=(1+\alpha) b^{\prime}, \\
f=(1-\beta) a, & g=(1+\beta) b .
\end{array}
$$

In order to avoid omitting important features outside the classical domain range $[a, b]$, the extension rate $\beta$ is proposed to obtain the joint domain. The joint domain $[f, g]$ is obtained by (6). Finally, the obtained classical domain $X_{o}=[a, b]$ and joint domain $X=[f, g]$ are two intervals in the real number 
TABLE 2: Matter-element model.

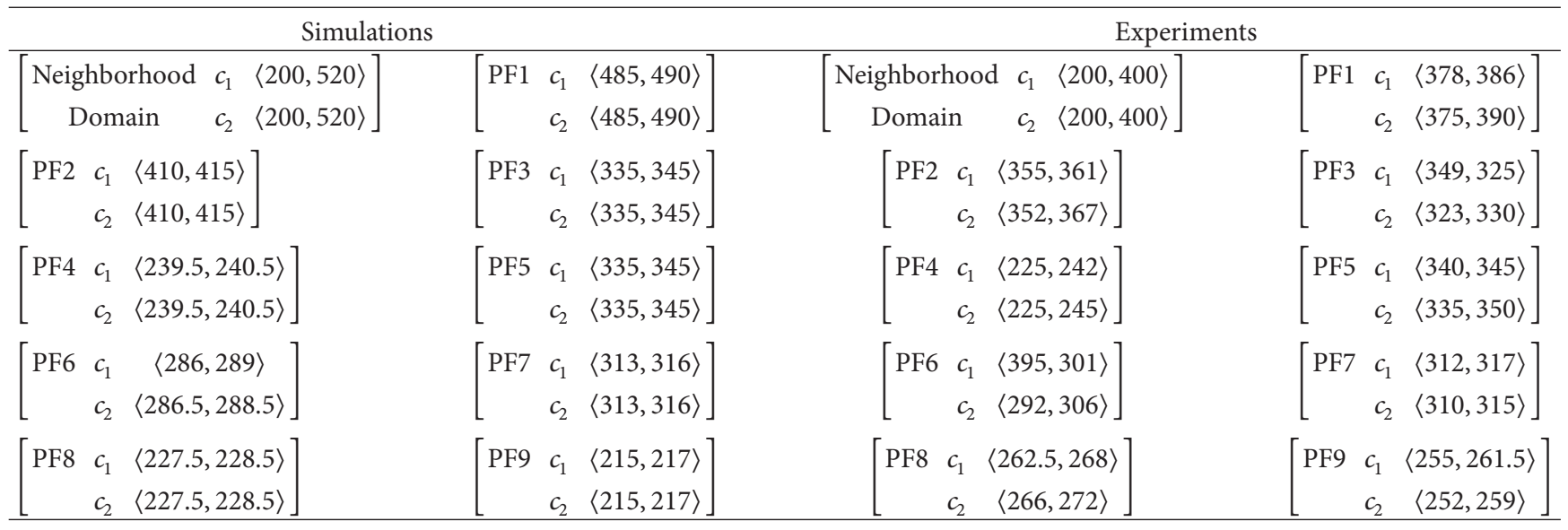

TABLE 3: Simulated fault diagnosis results.

\begin{tabular}{lcccccccccc}
\hline Qty & $\mathrm{ED}_{\mathrm{PF} 1}$ & $\mathrm{ED}_{\mathrm{PF} 2}$ & $\mathrm{ED}_{\mathrm{PF} 3}$ & $\mathrm{ED}_{\mathrm{PF} 4}$ & $\mathrm{ED}_{\mathrm{PF} 5}$ & $\mathrm{ED}_{\mathrm{PF} 6}$ & $\mathrm{ED}_{\mathrm{PF} 7}$ & $\mathrm{ED}_{\mathrm{PF} 8}$ & $\mathrm{ED}_{\mathrm{PF9}}$ & $\mathrm{Results}$ \\
\hline 1 & $\mathbf{1 . 0 0 0}$ & -0.585 & -0.839 & -0.981 & -0.722 & -0.931 & -0.893 & -0.991 & -1.000 & $\mathrm{PF} 1$ \\
2 & $\mathbf{1 . 0 0 0}$ & -0.585 & -0.839 & -0.981 & -0.722 & -0.931 & -0.983 & -0.991 & -1.000 & $\mathrm{PF} 1$ \\
3 & -0.186 & $\mathbf{1 . 0 0 0}$ & -0.224 & -0.910 & -0.337 & -0.670 & -0.485 & -0.958 & -1.000 & $\mathrm{PF} 2$ \\
4 & -0.176 & $\mathbf{1 . 0 0 0}$ & -0.224 & -0.910 & 0.337 & -0.670 & -0.485 & -0.958 & -1.000 & $\mathrm{PF} 2$ \\
5 & -1.000 & -0.357 & $\mathbf{1 . 0 0 0}$ & -0.549 & -0.004 & 0.054 & 0.564 & -0.662 & -0.760 & PF3 \\
6 & -1.000 & -0.357 & $\mathbf{1 . 0 0 0}$ & -0.549 & -0.004 & 0.054 & 0.565 & -0.662 & -0.759 & PF3 \\
7 & -1.000 & -0.885 & -0.642 & $\mathbf{1 . 0 0 0}$ & -0.822 & -0.258 & -0.510 & 0.982 & 0.465 & PF4 \\
8 & -1.000 & -0.885 & -0.642 & $\mathbf{1 . 0 0 0}$ & -0.822 & -0.258 & -0.510 & 0.987 & 0.468 & PF4 \\
9 & -0.584 & 0.406 & 0.366 & -0.843 & $\mathbf{1 . 0 0 0}$ & -0.420 & -0.093 & -0.926 & -1.000 & PF5 \\
10 & -0.584 & 0.406 & 0.369 & -0.842 & $\mathbf{1 . 0 0 0}$ & -0.419 & -0.091 & -0.926 & -1.000 & PF5 \\
11 & -1.000 & -0.633 & 0.140 & -0.007 & -0.432 & $\mathbf{1 . 0 0 0}$ & 0.562 & -0.162 & -0.289 & PF6 \\
12 & -1.000 & -0.631 & 0.147 & -0.007 & -0.429 & $\mathbf{1 . 0 0 0}$ & 0.571 & -0.161 & -0.289 & PF6 \\
13 & -1.000 & -0.530 & 0.459 & -0.170 & -0.273 & 0.648 & $\mathbf{1 . 0 0 0}$ & -0.308 & -0.424 & PF7 \\
14 & -1.000 & -0.530 & 0.459 & -0.176 & -0.273 & 0.636 & $\mathbf{1 . 0 0 0}$ & -0.313 & -0.429 & PF7 \\
15 & -1.000 & -0.893 & -0.668 & 0.855 & -0.834 & -0.312 & -0.545 & $\mathbf{1 . 0 0 0}$ & 0.455 & PF8 \\
16 & -1.000 & -0.895 & -0.668 & 0.855 & -0.834 & -0.322 & -0.552 & $\mathbf{1 . 0 0 0}$ & 0.450 & PF8 \\
17 & -1.000 & -0.934 & -0.796 & 0.136 & -0.898 & -0.578 & -0.721 & 0.711 & $\mathbf{1 . 0 0 0}$ & PF9 \\
18 & -1.000 & -0.932 & -0.791 & 0.169 & -0.896 & -0.566 & -0.713 & 0.761 & $\mathbf{1 . 0 0 0}$ & PF9 \\
\hline & & & & & & & &
\end{tabular}

field $[-\infty, \infty]$. If $x$ is a point of real number, the correlation function is defined as

$$
K(x)= \begin{cases}-\rho\left(x, X_{o}\right), & x \in X_{o}, \\ \frac{\rho\left(x, X_{o}\right)}{\rho(x, X)-\rho\left(x, X_{o}\right)}, & x \notin X_{o} .\end{cases}
$$

Figure 2 is the chaotic signal synchronization and extension diagnostic system flow chart. First, the measured voltage of solar photovoltaic system is recorded, and then the recorded voltage signal to be measured is imported into the slave system of chaotic signal synchronization system. The chaotic synchronization system generates the chaotic dynamic error signal after the subtraction between master system and slave system. The kinematic trajectory formed of chaotic dynamic error signal is the main basis of extension diagnosis, which is the fault feature of solar photovoltaic system. Finally, as long as the chaotic dynamic error signal is imported into the finished extension matter-element model, the fault category can be identified rapidly and accurately by extension diagnosis of fault conditions.

\section{Simulation and Experimental Results}

The solar photovoltaic system was simulated by using MATLAB 2009 a. The solar photovoltaic array for experiment was Agilent Technologies E4360A modularized solar array simulator platform. The solar panel was SM 1611. The specifications are as follows: open-circuit voltage is $3 \mathrm{~V}$, short-circuit current is $0.8 \mathrm{~A}$, maximum power point voltage is $2.36 \mathrm{~V}$, maximum power point current is $0.72 \mathrm{~A}$, solar illumination is 
TABLE 4: Actual fault diagnosis results.

\begin{tabular}{lcccccccccc}
\hline Qty & $\mathrm{ED}_{\mathrm{PF} 1}$ & $\mathrm{ED}_{\mathrm{PF} 2}$ & $\mathrm{ED}_{\mathrm{PF} 3}$ & $\mathrm{ED}_{\mathrm{PF} 4}$ & $\mathrm{ED}_{\mathrm{PF} 5}$ & $\mathrm{ED}_{\mathrm{PF} 6}$ & $\mathrm{ED}_{\mathrm{PF} 7}$ & $\mathrm{ED}_{\mathrm{PF} 8}$ & $\mathrm{ED}_{\mathrm{PF} 9}$ & $\mathrm{Results}$ \\
\hline 1 & $\mathbf{1 . 0 0 0}$ & -0.391 & -0.790 & -1.000 & -0.641 & -0.887 & -0.843 & -0.963 & -0.978 & $\mathrm{PF} 1$ \\
2 & $\mathbf{1 . 0 0 0}$ & -0.391 & -0.790 & -1.000 & -0.641 & -0.887 & -0.843 & -0.963 & -0.978 & $\mathrm{PF} 1$ \\
3 & -0.222 & $\mathbf{1 . 0 0 0}$ & -0.476 & -1.000 & -0.103 & -0.719 & -0.610 & -0.908 & -0.945 & $\mathrm{PF} 2$ \\
4 & -0.222 & $\mathbf{1 . 0 0 0}$ & -0.476 & -1.000 & -0.103 & -0.719 & -0.610 & -0.907 & -0.945 & PF2 \\
5 & -0.743 & -0.391 & $\mathbf{1 . 0 0 0}$ & -1.000 & -0.042 & -0.115 & 0.226 & -0.710 & -0.829 & PF3 \\
6 & -0.743 & -0.392 & $\mathbf{1 . 0 0 0}$ & -1.000 & -0.044 & -0.116 & 0.224 & -0.710 & -0.829 & PF3 \\
7 & -1.000 & -0.964 & -0.890 & $\mathbf{1 . 0 0 0}$ & -0.931 & -0.787 & -0.858 & -0.581 & -0.448 & PF4 \\
8 & -0.488 & -0.964 & -0.890 & $\mathbf{1 . 0 0 0}$ & -0.931 & -0.787 & -0.858 & -0.581 & -0.448 & PF4 \\
9 & -0.488 & -0.074 & -0.205 & -1.000 & $\mathbf{1 . 0 0 0}$ & -0.573 & -0.408 & -0.860 & -0.917 & PF5 \\
10 & -1.000 & -0.073 & -0.205 & -1.000 & $\mathbf{1 . 0 0 0}$ & -0.573 & -0.408 & -0.860 & -0.917 & PF5 \\
11 & -1.000 & -0.817 & -0.440 & -0.812 & -0.653 & $\mathbf{1 . 0 0 0}$ & -0.280 & -0.517 & -0.638 & PF6 \\
12 & -0.888 & -0.817 & -0.440 & -0.821 & -0.653 & $\mathbf{1 . 0 0 0}$ & -0.280 & -0.517 & -0.638 & PF6 \\
13 & -0.888 & -0.572 & 0.145 & -1.000 & -0.272 & -0.017 & $\mathbf{1 . 0 0 0}$ & -0.678 & -0.810 & PF7 \\
14 & -1.000 & -0.572 & 0.145 & -1.000 & -0.272 & -0.017 & $\mathbf{1 . 0 0 0}$ & -0.678 & -0.810 & PF7 \\
15 & -1.000 & -0.865 & -0.589 & -0.074 & -0.745 & -0.205 & -0.471 & $\mathbf{1 . 0 0 0}$ & 0.363 & PF8 \\
16 & -1.000 & -0.865 & -0.589 & -0.074 & -0.745 & -0.205 & -0.472 & $\mathbf{1 . 0 0 0}$ & 0.363 & PF8 \\
17 & -1.000 & -0.889 & -0.662 & 0.015 & -0.791 & -0.346 & -0.566 & 0.285 & $\mathbf{1 . 0 0 0}$ & PF9 \\
18 & -1.000 & -0.889 & -0.662 & 0.015 & -0.791 & -0.346 & -0.565 & 0.286 & $\mathbf{1 . 0 0 0}$ & PF9 \\
\hline & & & & & & & & &
\end{tabular}

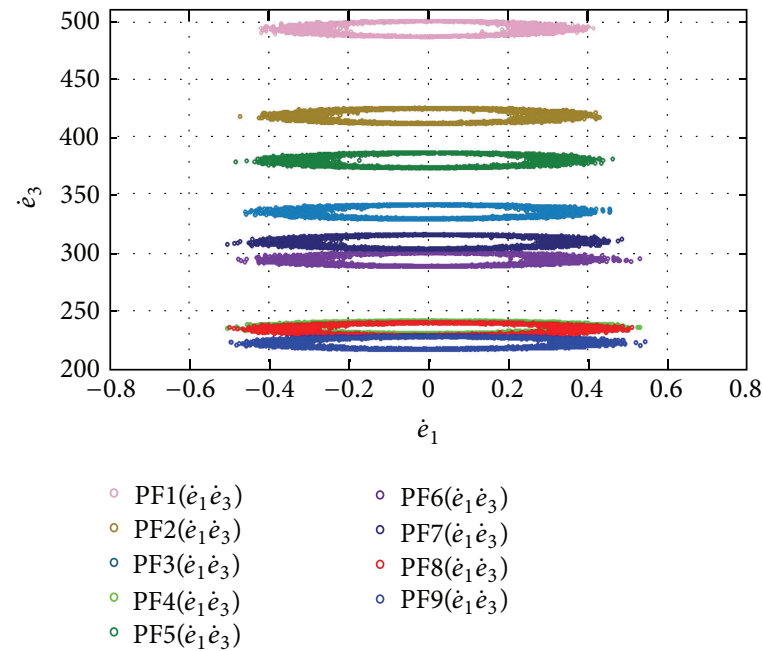

(a)

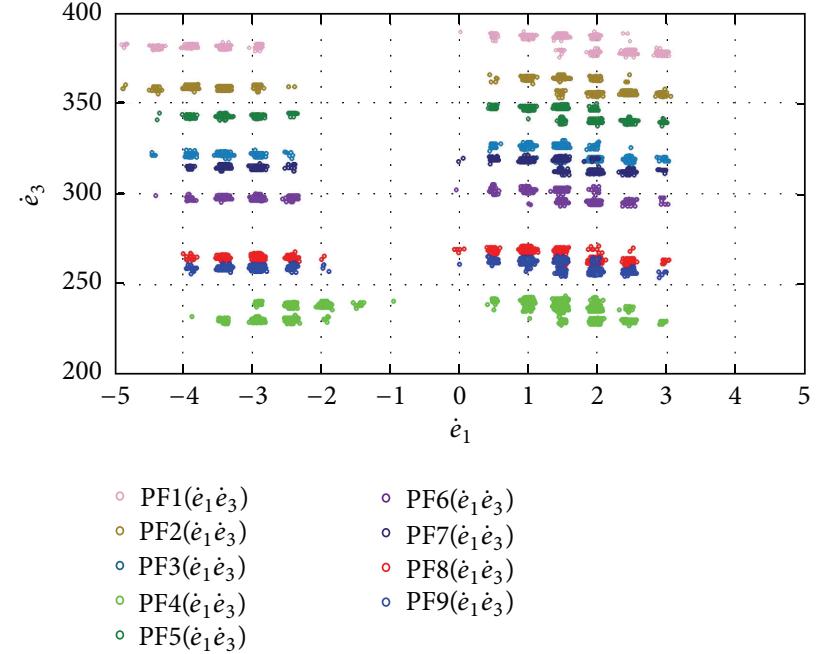

(b)

FIGURE 3: (a) $\dot{e}_{1} \dot{e}_{3}$ dynamic error plane of nine simulated fault conditions. (b) $\dot{e}_{1} \dot{e}_{3}$ dynamic error plane of nine physical fault conditions.

$1000 \mathrm{~W} / \mathrm{m}^{2}$, ambient temperature is $25^{\circ} \mathrm{C}$, and the connection mode is 10 series and 2 parallel. The fault types are shown in Table 1. The voltage-current characteristic curve with faults was captured and recorded, and the voltage in nine cases was imported into the chaotic synchronization system, so that the chaotic synchronization system generated dynamic error. Figure 3(a) shows the $\dot{e}_{1} \dot{e}_{3}$ dynamic error plane of nine simulated fault conditions. Figure 3 (b) shows the $\dot{e}_{1} \dot{e}_{3}$ dynamic error plane of nine actual fault conditions. The fault features are extracted as the base of extension diagnosis. Finally, the matter-element models are finished in Table 2 for fault recognition. Finally, 18 data are imported for the system to distinguish. There are 18 data imported into simulated and actual diagnoses, respectively. The diagnostic results are shown in Tables 3 and 4 . They show that the proposed scheme has good diagnostic performance.

\section{Conclusion}

This study diagnosed the faults in solar photovoltaic system based on chaotic signal synchronization and used extension theory to distinguish the fault category. As the chaotic 
synchronization allows the system to have obvious nonlinear motion due to the change in system parameter, this obvious kinematic trajectory was used as the characteristic values of faults, and excellent diagnosis effect was obtained by the intelligent recognition of extension. The extension theory does not need iteration or learning, and its diagnosis is very fast in comparison to general neural diagnosis. Accurate, rapid, timesaving, and cost saving fault recognition could be achieved by capturing one voltage signal and two characteristic values of chaos. The cost was much lower than other traditional methods which need many sensors to capture physical quantities for distinguishing faults. As an intelligent fault diagnostor, its effect is excellent.

\section{Conflict of Interests}

The authors declare that they have no conflict of interests.

\section{Acknowledgments}

The author would like to thank Professor Her-Terng Yau for his valuable comments on chaos synchronization.

\section{References}

[1] Y. Wu, Q. Lan, and Y. Sun, "Application of BP neural network fault diagnosis in solar photovoltaic system," in Proceedings of the 2009 IEEE International Conference on Mechatronics and Automation (ICMA '09), pp. 2581-2585, August 2009.

[2] M. H. Wang and C. P. Hung, "Extension neural network and its applications," Neural Networks, vol. 16, no. 5-6, pp. 779-784, 2003.

[3] H. Patel and V. Agarwal, "MATLAB-based modeling to study the effects of partial shading on PV array characteristics," IEEE Transactions on Energy Conversion, vol. 23, no. 1, pp. 302-310, 2008.

[4] K. H. Chao, S. H. Ho, and M. H. Wang, "Modeling and fault diagnosis of a photovoltaic system," Electric Power Systems Research, vol. 78, no. 1, pp. 97-105, 2008.

[5] M. Veerachary, "PSIM circuit-oriented simulator model for the nonlinear photovoltaic sources," IEEE Transactions on Aerospace and Electronic Systems, vol. 42, no. 2, pp. 735-740, 2006.

[6] K. Ding, X. G. Bian, H. H. Liu, and T. Peng, "A MATLABsimulink-based PV module model and its application under conditions of nonuniform irradiance," IEEE Transactions on Energy Conversion, vol. 27, no. 4, pp. 864-872, 2012.

[7] N. Onat, "Recent developments in maximum power point tracking technologies for photovoltaic systems," International Journal of Photoenergy, vol. 2010, Article ID 245316, 11 pages, 2010.

[8] C. H. Huang, C. H. Lin, and C. L. Kuo, "Chaos synchronizationbased detector for power-quality disturbances classification in a power system," IEEE Transactions on Power Delivery, vol. 26, no. 2, pp. 944-953, 2011.

[9] W. H. Deng and C. P. Li, "Chaos synchronization of the fractional Lü system,” Physica A, vol. 353, no. 1-4, pp. 61-72, 2005.

[10] C. Li and G. Peng, "Chaos in Chen's system with a fractional order," Chaos, Solitons and Fractals, vol. 22, no. 2, pp. 443-450, 2004.
[11] A. K. Golmankhaneh, R. Arefi, and D. Baleanu, “The proposed modified Liu system with fractional order," Advances in Mathematical Physics, vol. 2013, Article ID 186037, 6 pages, 2013.

[12] A. K. Golmankhaneh, R. Arefi, and D. Baleanu, "Synchronization in a nonidentical fractional order of a proposed modified system," Journal of Vibration and Control, 2013.

[13] D. Baleanu, K. Diethelm, E. Scalas, and J. J. Trujillo, Fractional Calculus Models and Numerical Methods, Series on Complexity, Nonlinearity and Chaos, World Scientific, Singapore, 2012.

[14] S. Y. Xiang, W. Pan, B. Luo et al., "Synchronization of unpredictability-enhanced chaos in VCSELs with variable-polarization optical feedback," IEEE Journal of Quantum Electronics, vol. 47, no. 10, pp. 1354-1361, 2011.

[15] C. T. Hsieh, M. H. Wang, and Y. P. Kuo, "PEM fuel cell diagnostic system using the extension theory," International Journal of Distributed Sensor Networks, vol. 2013, Article ID 539250, 7 pages, 2013. 


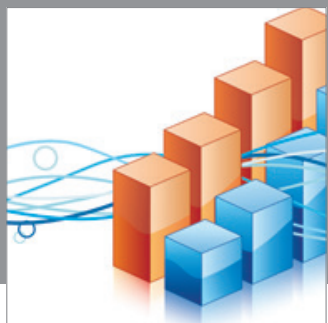

Advances in

Operations Research

mansans

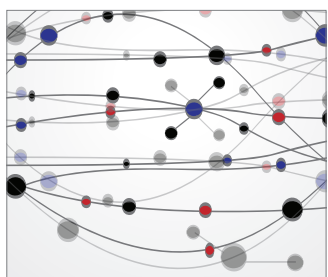

The Scientific World Journal
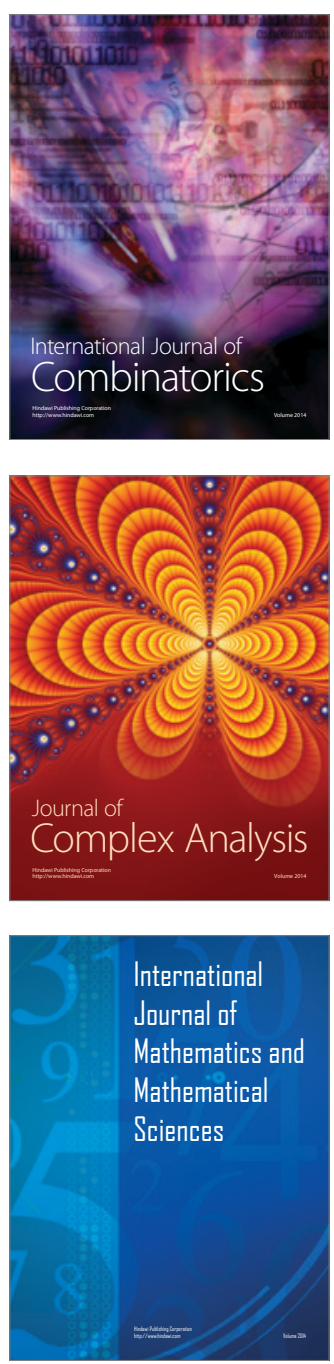
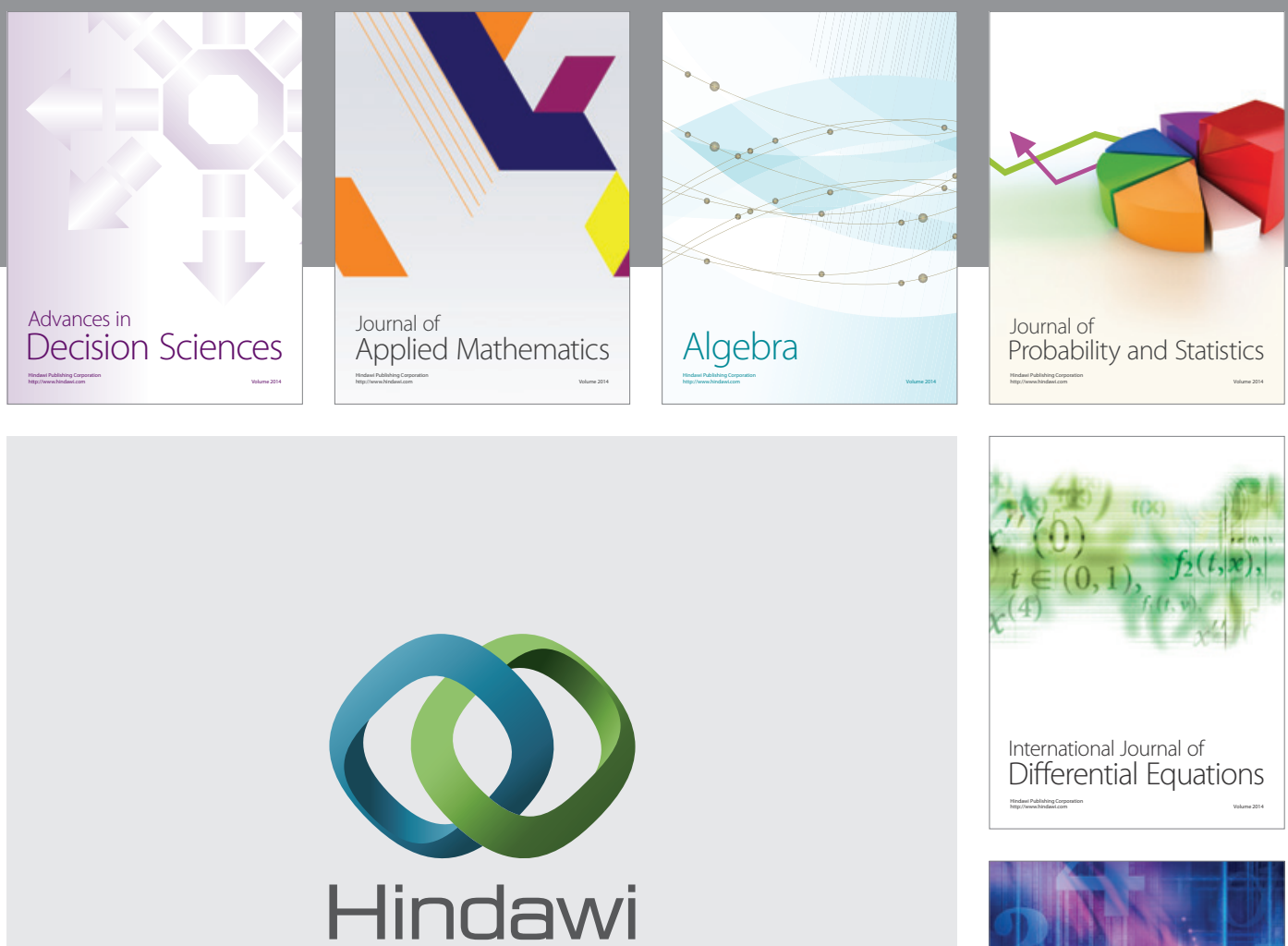

Submit your manuscripts at http://www.hindawi.com
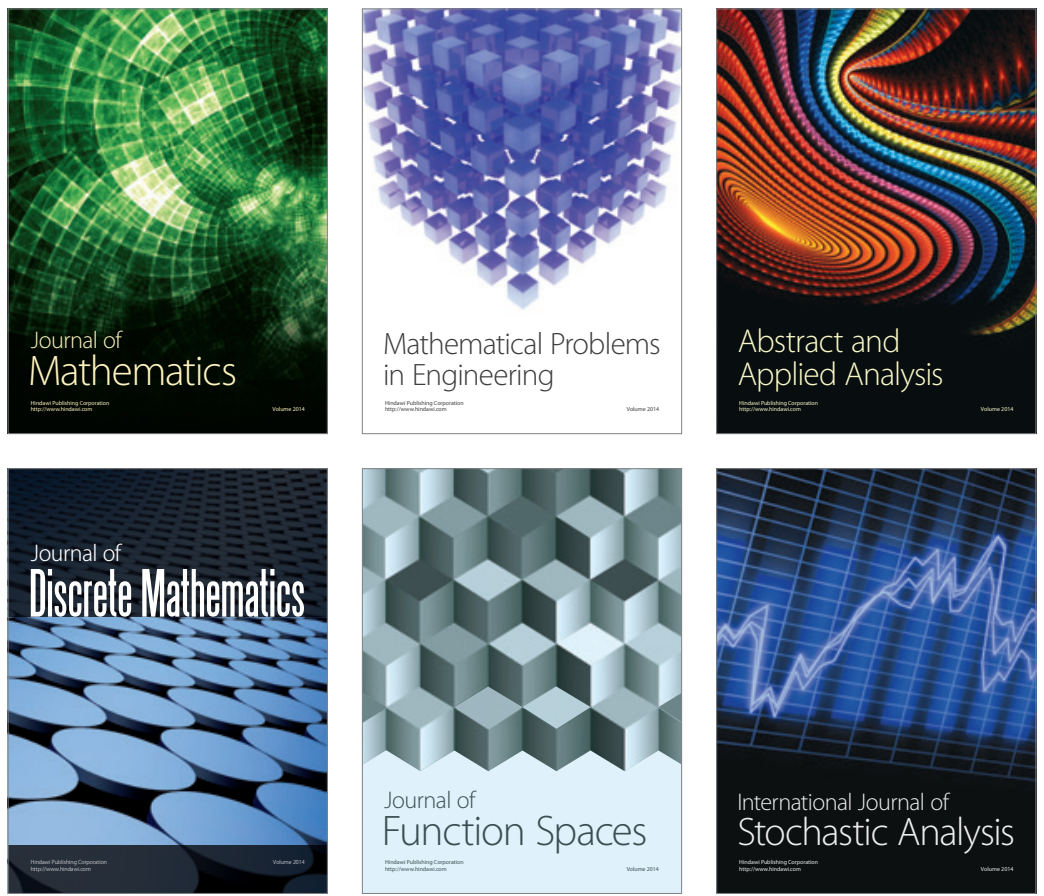

Journal of

Function Spaces

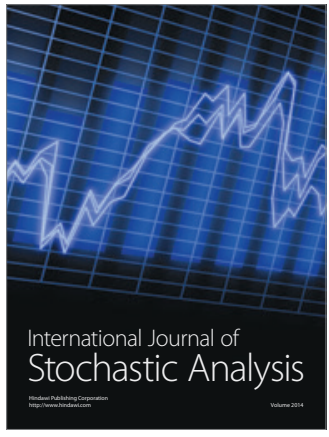

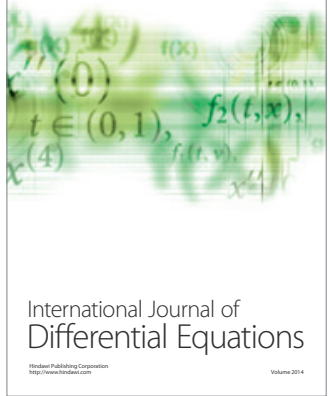
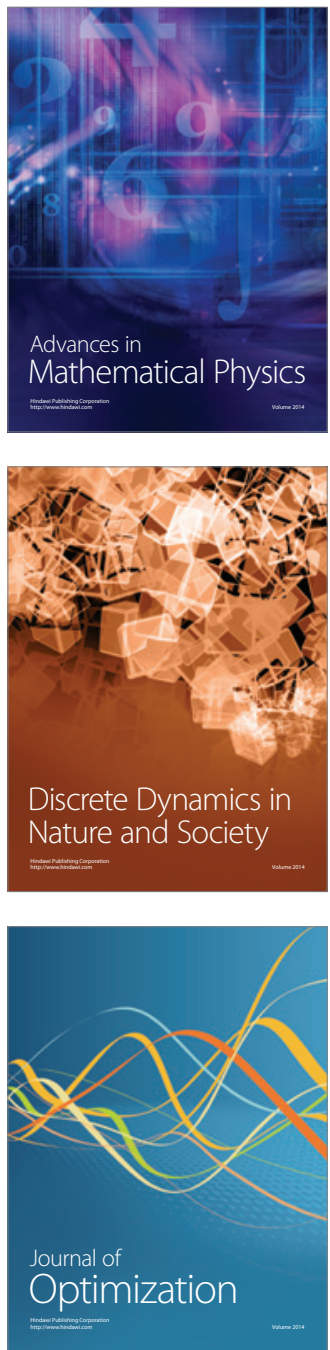